\title{
12-0-tetradecanoylphorbol-1,3-acetate-induced degradation of protein kinase B via ubiquitin- -proteasomal pathway depends on its Ser473 phosphorylation in gastric cancer cells
}

\author{
Bing Zhang ${ }^{1}$, Chun Xia ${ }^{2}$ \\ ${ }^{1}$ Medical School, Xiamen University, Fujian, China \\ ${ }^{2}$ Zhongshan Hospital, Xiamen University, Fujian, China
}

\begin{abstract}
TPA (12-O-tetradecanoylphorbol-1, 3-acetate) can induce cell apoptosis and cause PKB (protein kinase $\mathrm{B}$ ) degradation correlated with its phosphorylation in gastric cancer cells. We investigated whether the ubiquitin-proteasomal pathway is involved in TPA-induced PKB degradation. The results showed that TPA could induce PKB ubiquitination by inhibiting its phosphorylation at the serine 473 site. Moreover, MG132 (26S proteasome inhibitor) partially inhibited TPA-induced degradation of PKB. Taken together, TPA could degrade PKB via the ubiquitin-proteasomal pathway, and the suppression of PKB phosphorylation at the serine 473 site might be a prerequisite for the TPA-induced ubiquitination in gastric cancer cells. (Folia Histochemica et Cytobiologica 2013, Vol. 51, No. 1, 11-17)
\end{abstract}

Key words: Akt; PKB, phosphorylation, ubiquitin-proteasomal pathway, gastric cancer cells

\section{Introduction}

TPA (12-O-tetradecanoylphorbol-1, 3-acetate), a phorbol ester, can be found in many Chinese medicinal herbs and food types. Some evidence supports TPA as a tumor promoter agent [1]. However, other authors and ourselves showed that TPA could induce apoptosis in some cell lines, such as skin epidermal JB6 cells, gastric cancer cells and prostate cancer cells [2-4]. Therefore, biological effects of TPA can differ depending on the cell type.

PKB (protein kinase B, also known as Akt) is a serine-threonine kinase activated by extracellular factors through a phosphorylation mechanism [5]. It contains three conserved phosphorylation sites, which are Thr308 in the activation loop, Thr450 in the turn

Correspondence address: C. Xia,

Zhongshan Hospital, Xiamen University, Fujian, China;

tel.:+592 29930 80; fax:+86 59259214 61;

e-mail: chunxia@xmu.edu.cn motif (TM), and Ser473 in the hydrophobic motif(HM). Upon growth factor stimulation, the phosphatidylinositol 3-kinase (PI3K) produces phosphatidylinositol 3,4,5-trisphosphate $(\operatorname{Ptd} \operatorname{In}(3,4,5) \mathrm{P} 3$, PIP3) that recruits $\mathrm{PKB}$ and 3-phosphoinositide-dependent protein kinase (PDK1) to the plasma membrane through their pleckstrin homology (PH) domain. At the plasma membrane, PDK1 phosphorylates PKB at Thr308 to activate $\mathrm{PKB}[5,6]$. In addition to the Thr308 phosphorylation, $\mathrm{PKB}$ is phosphorylated at Ser473 in HM, which further enhances PKB activity [7, 8]. Various types of human cancers have been found to harbor hyperactivated $\mathrm{PKB}$, and the activated $\mathrm{PKB}$ phosphorylates a variety of proteins involved in tumorigenesis and drug resistance of cancer cells [9-11]. Thus, inhibiting PKB activity has gradually become the effective way for cancer therapy $[12,13]$. Previously, we reported that TPA could induce PKB degradation and inhibit the activity of PKB, followed by apoptosis in gastric cancer cells [4], however, the mechanism of PKB degradation by TPA was unclear. 
Many studies elucidated the mechanisms of signaling proteins' degradation, such as proteasomedependent degradation and lysosomal pathway [14]. During proteasome-dependent degradation, at first proteins are usually ubiquitinated by a complex containing ubiquitin-activating enzyme (E1), ubiquitinconjugating enzyme (E2), and ubiquitin-protein ligase (E3). Then, the ubiquitinated protein is recognized by the $26 \mathrm{~S}$ proteasome and targeted for protein degradation [14]. Moreover, in certain cases, phosphorylation promotes ubiquitination and degradation of signaling proteins, such as IkB and b-catenin [15]. Recently, PKB was shown to undergo ubiquitination when cells were treated with growth factors or cytokines known to induce PKB activation [16]. Thus, the ubiquitination and phosphorylation of PKB might play an important role in its degradation induced by TPA in gastric cancer cells.

Here, we used different inhibitors and expression vector transfection to investigate whether in cells exposed to TPA, PKB is ubiquitinated and targeted to the proteasome, where it is degraded. The results showed that PKB ubiquitination was associated with the inhibition of its phosphorylation at the serine 473 site. TPA-induced ubiquitination of PKB was suppressed by the mutation of the serine 473 site of PKB, but not the threonine 308 site. Even after treatment with MG132 (26S proteasome inhibitor), the ubiquitination of $\mathrm{PKB}$ could not be observed in cells transfected by the vector carrying Ser473 point mutant of PKB. Thus, TPA effectively inhibited PKB activity and caused PKB degradation through the ubiquitin-proteasomal pathway, which was associated with the repression of PKB phosphorylation at Ser473, but not Thr308, in gastric cancer cells.

\section{Material and methods}

Cell culture condition and transfection. Human embryonic kidney 293T cell line (obtained from ATCC, CRL-11268, USA) was maintained in DMEM medium, and gastric cancer cell line BGC-823 (purchased from the Institute of Cell Biology, Shanghai, China) was cultured in RPMI-1640 medium that contained $10 \%$ fetal bovine serum, $1 \mathrm{mM}$ glutamine, and $100 \mathrm{mg} / \mathrm{mL}$ penicillin. Cells were transfected with different expression vectors such as His-Ub, MycPKB, HA-PKB/T308A, and HA-PKB/S473A (supplied by Dr. Qiao Wu) using the Ca3(PO4)2 sedimentation method in all the experiments, as previously described [17]. Briefly, cells were cultured in a $60 \mathrm{~mm}$ dish for $20-24 \mathrm{~h}$, after which the plasmid-containing buffer (double distilled water: $220 \mu \mathrm{L}$, plasmid: $2 \mathrm{mg}, 2.5 \mathrm{M} \mathrm{CaCl} 2: 25 \mathrm{~mL}, 2 \mathrm{xHBS}: 250 \mathrm{~mL}$ ) was added for $24 \mathrm{~h}$, followed by different experimental procedures.
Antibodies and inhibitors. Monoclonal antibodies against PKB (anti-human), P-PKB-Ser473 (anti-human), P-PKBThr308 (anti-human), and ubiquitin (anti-human) were purchased from Cell Signaling Technology (Beverly, USA). Tubulin (anti-human) was purchased from Santa Cruz (Santa Cruz Biotechnology Co, Shanghai, China). TPA, the proteasome inhibitors, ALLN ( $N$-acetyl-Leu-Leu-Norleu-al), MG132 ( $N$-Cbz-Leu-Leu-Leu-al), and ALLM ( $N$-acetylLeu-Leu-Met-al) were purchased from Sigma-Aldrich (St. Louis, MO, USA).

Immunoprecipitation. Cells were treated with TPA (100 ng/ $/ \mathrm{mL}$ ) for $24 \mathrm{hr}$ or $48 \mathrm{hr}$, and then harvested. Cells were lysed in lysis buffer (140 mM NaCl, 0.5\% NP40, $100 \mathrm{mM} \mathrm{NaF}$, $50 \mathrm{mM}$ Tris/ $\mathrm{HCl} \mathrm{pH} \mathrm{8.0),} \mathrm{then} \mathrm{vortexed} \mathrm{and} \mathrm{centrifuged} \mathrm{at}$ $30000 \mathrm{~g} 4^{\circ} \mathrm{C}$ for 30 mins to obtain all cell lysates (supernatant). The lysates were incubated with anti-PKB for $1 \mathrm{hr}$, and were subsequently incubated with protein A-sepharose beads (Sigma, St. Louis, USA) for additional $4 \mathrm{~h}$ at $4^{\circ} \mathrm{C}$ as described previously [17]. After a brief centrifugation at $1000 \mathrm{~g}$, the lysates (immunoprecipitate) were collected and divided into two parts. One was subjected to Western blot analysis as described below with corresponding antibodyubiquitin to indicate the interaction with PKB. The other was analyzed by Western blotting as described below with the anti-PKB antibody to indicate the amount of immunoprecipitated PKB protein used in each lane.

Detection of ubiquitin modification in cell culture. BGC823 cells and HEK293T cells transfected with different expression vectors (His-Ub, Myc-PKB, HA-PKB/T308A, and HA$\mathrm{PKB} / \mathrm{S} 473 \mathrm{~A}$ ) were pretreated with the inhibitor (MG132) of $26 \mathrm{~S}$ proteasome for $3 \mathrm{~h}$. Cells were harvested following incubation with TPA (100 ng/mL) for $24 \mathrm{~h}$. As previously described [19], cells were washed twice with PBS and lysed by adding Buffer A $(6 \mathrm{~mol} / \mathrm{L}$ guanidinium- $\mathrm{HCl}, 0.1 \mathrm{M}$ $\mathrm{Na}_{2} \mathrm{HPO}_{4} / \mathrm{NaH}_{2} \mathrm{PO}_{4}, 5 \mathrm{mM}$ imidazole, $10 \mathrm{mM} \beta$-mercaptoethanol and $0.01 \mathrm{M}$ Tris- $\mathrm{HCl}, \mathrm{pH} 8.0)$. Then, a fraction of the lysates was analyzed by Western blotting analysis (as described below) with an antibody to a tag present on an expressed substrate, such as HA and Myc. The remainder of the lysates was incubated with $50 \mathrm{ml}$ of $\mathrm{Ni}^{2+}$-NTA-agarose beads prewashed by lysis buffer, and incubated for $2 \mathrm{~h}$ at room temperature. Beads were harvested with centrifugation at $660 \times g$ and washed with the following solutions: Buffer B (6 M guanidinium- $\mathrm{HCl}, 0.1 \mathrm{M} \mathrm{Na} 2 \mathrm{HPO} 4 / \mathrm{NaH}_{2} \mathrm{PO}_{4}$, $10 \mathrm{mM}$ b-mercaptoethanol and 0.01 M Tris- $\mathrm{HCl}, \mathrm{pH} 8.0$ ); Buffer C (8 M Urea, 0.1 M Na $\mathrm{HPO}_{4} / \mathrm{NaH}_{2} \mathrm{PO}_{4}, 10 \mathrm{mM}$ $\beta$-mercaptoethanol, and 0.01 M Tris- $\mathrm{HCl}, \mathrm{pH}$ 8.0) and Buffer D (8M Urea, 0.1 M Na2HPO4/NaH2PO4, 10 mM b-mercaptoethanol, and $0.01 \mathrm{M}$ Tris- $\mathrm{HCl}, \mathrm{pH} 6.3$ plus $0.2 \%$ Triton X-100). Then, the beads were eluted with $200 \mathrm{mM}$ imidazole in 5\% SDS, $0.15 \mathrm{M}$ Tris- $\mathrm{HCl}, \mathrm{pH}$ 6.7, 30\% glycerol and $0.72 \mathrm{M} \beta$-mercaptoethanol. The eluates were subjected 
to Western blotting analysis (as described below) with an antibody to a tag present on an expressed substrate, such as HA and Myc.

Western blot analysis. Protein extracts were electrophoresed on $10 \%$ denaturing gel and electroblotted onto a nitrocellulose membrane. Each membrane was subsequently blocked with $5 \%$ dry non-fat milk in TBS-T (Tris-buffered saline with $2.5 \%$ Tween 20 ) and then incubated with primary antibodies against: PKB, P-PKB-Ser473, P-PKB-Thr308, Tubulin, Ubiquitin, Myc, and HA, as previously described [17]. The membranes were washed with TBS-T and incubated with secondary antibodies (Santa Cruz Biotechnology Co, Shanghai, China) conjugated with peroxidase, and the signal was detected by the chemiluminescent detection system (Pierce, Rockford, IL, USA) according to the manufacturer's instructions.

Statistical analysis. Descriptive characteristics are expressed as mean values \pm standard deviation $( \pm$ SD). One-way ANOVA was used to evaluate the statistical differences between the means of paired sets of data.

\section{Results}

\section{The ubiquitin-proteasomal pathway mediates TPA-induced PKB degradation}

Previously, we found that TPA significantly down-regulates the expression and activity of PKB in a timedependent manner in gastric cancer cells [4]. To reveal the pathway that mediates the TPA-induced PKB degradation, different inhibitors for protecting protein from degradation were used to treat BGC-823 cells in combination with TPA. The inhibitors were: MG132 (potent cell permeable proteasome inhibitor), ALLN (inhibitor for calpains, cathepsins and proteasomal proteolysis), and ALLM (a potential inhibitor for calpains and cathepsins, but not for proteasome). Western blotting analysis showed that MG132 and ALLN, but not ALLM, significantly inhibited TPAinduced PKB degradation, indicating that only the proteasome pathway was involved in PKB degradation (Figure 1A).

Binding of protein to ubiquitin is a prerequisite for its degradation via the proteasome pathway. Here, we investigated whether the degradation of PKB was associated with ubiquitination. After BGC-823 cells were treated with TPA for $24 \mathrm{~h}$ or $48 \mathrm{~h}$, the immunoprecipitation/Western blotting analysis revealed that TPA strongly enhanced the targeting of endogenous ubiquitin to PKB molecules in BGC-823 cells, as the smeared bands of $\mathrm{PKB}$, exhibiting the ubiquitination of PKB, could be clearly observed (Figure 1B). Simi- larly, when $293 \mathrm{~T}$ cells were co-transfected with ubiquitin and PKB expression vectors, ubiquitination of PKB upon TPA treatment was also observed, regardless of the absence or presence of MG132 (Figure 1C). MG132 enhanced the TPA-induced ubiquitination of PKB molecules in cells co-transfected with $\mathrm{PKB}$ and ubiquitin vectors (Figure 1C). Taken together, we concluded that the ubiquitin-proteasomal pathway mediates the TPA-induced PKB degradation.

\section{The ubiquitin-proteasomal pathway of PKB degradation induced by TPA is associated with PKB phosphorylation}

Since the phosphorylation status of PKB is an indicator of its activation, and the phosphorylation of two PKB residues, Thr308 and Ser473 (Figure 2A), is required for maximal PKB activation [6-8], we monitored PKB phosphorylation upon TPA treatment by using two specific PKB-phospho antibodies in Western blotting analysis. As shown in Figure 2B, both Ser473- and Thr30-phosphorylated forms of PKB were detected in BGC-823 cells. Upon treatment with TPA, we found that phosphorylation of PKB Ser473 was dramatically reduced, whereas Thr308 phosphorylation of PKB remained unchanged (Figure 2B). Since TPA has an inhibitory effect on PKB expression and TPA-induced inhibition of Ser473 phosphorylation might be associated with PKB degradation [4], we further investigated whether repression of PKB phosphorylation by TPA was related to PKB ubiquitination and degradation. Two point mutants of PKB, in which Ser473 or Thr308 was replaced with Ala (PKB/S473A, PKB/T308A), were constructed and transfected into HEK293T cells. We observed that TPA induced ubiquitination of PKB in PKB/T308A expressing cells, but hardly any $\mathrm{PKB}$ ubiquitination could be detected in $\mathrm{PKB} / \mathrm{S} 473 \mathrm{~A}$ expressing cells even in the presence of MG132 (Figure 2C). In addition, we noted that MG132 still showed an increasing effect against TPA-induced ubiquitination of PKB in $\mathrm{PKB} / \mathrm{T} 308 \mathrm{~A}$ expressing cells, but not in $\mathrm{PKB} / \mathrm{S} 473 \mathrm{~A}$ expressing cells (Figure 2C). Clearly, these results showed that TPA-induced inhibition of phosphorylation at Ser473 of PKB was associated with ubiquitination and degradation of PKB.

\section{Discussion}

In this report, we identified that TPA could degrade PKB protein via the ubiquitin-proteasomal pathway, and PKB ubiquitination by TPA depended on the repression of $\mathrm{PKB}$ phosphorylation at the serine 473 site in gastric cancer cells. 
A
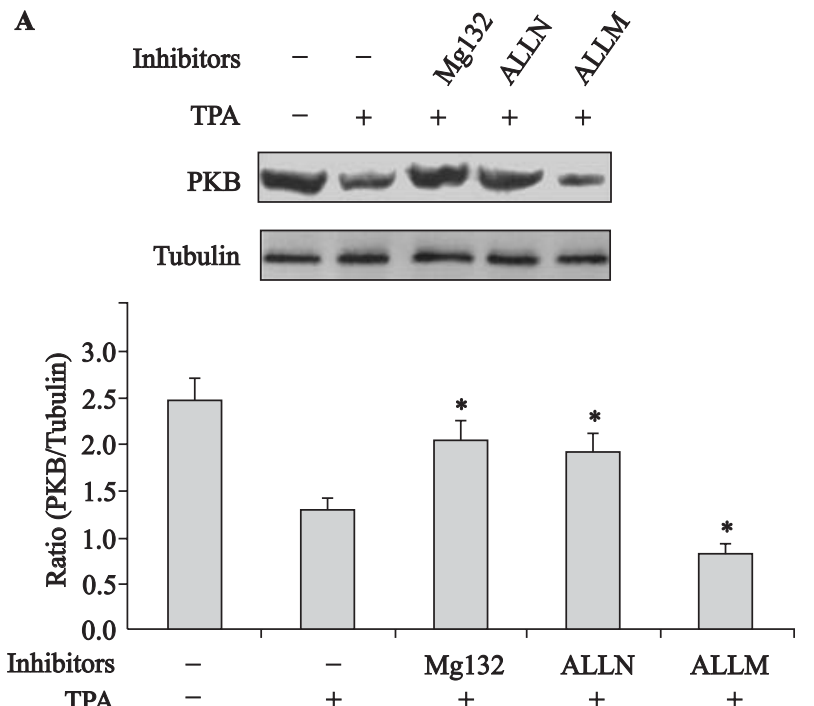

B
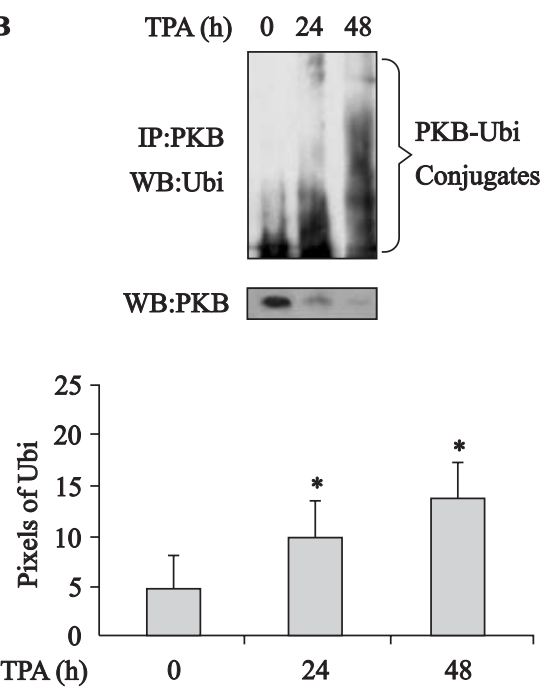
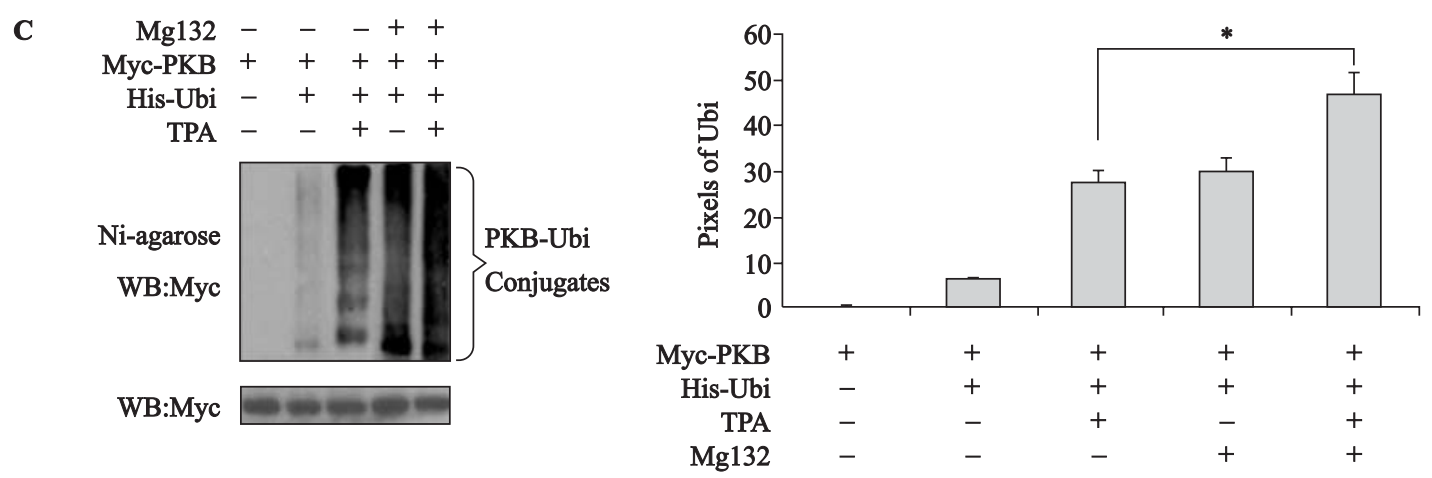

Figure 1. TPA-induced PKB degradation is mediated by the ubiquitin-proteasomal pathway. A. Effects of various inhibitors on degradation of PKB induced by TPA in gastric cancer BGC-823 cells. Cells were pretreated with MG132 (20 $\mu \mathrm{M})$, $\operatorname{ALLN}(25 \mu \mathrm{M})$ or ALLM $(25 \mu \mathrm{M})$ for $3 \mathrm{~h}$, followed by TPA treatment $(100 \mathrm{ng} / \mathrm{mL})$ for $24 \mathrm{~h}$. Cell lysates were prepared as described in Materials and Methods, and then analyzed by Western blotting with anti-PKB antibody. Tubulin expression served as loading control. B. TPA induces ubiquitination of PKB in gastric cancer BGC-823 cells. Cells were treated with TPA (100 ng/mL) for different time as indicated. Cells lysates were prepared as described in Materials and Methods, and then immunoprecipitated with anti-PKB antibody. Immunoprecipitates were separated electrophoretically and subjected to Western blotting analyses with anti-Ubiquitin and anti-PKB antibodies. C. TPA induced ubiquitination of PKB in HEK293T cells co-transfected with PKB and ubiquitin expression vectors. Cells were co-transfected with Myc-PKB and His-Ubiquitin expression vectors, then treated with MG132 $(20 \mu \mathrm{M})$ for $3 \mathrm{~h}$ and/or TPA $(100 \mathrm{ng} / \mathrm{mL})$ for $24 \mathrm{~h}$. Cell lysates were prepared as described in Materials and Methods. A fraction of the lysate was analyzed by Western blotting analysis with a Myc antibody (lower panel). The remainder of the lysate was incubated with Ni-agarose and bound proteins were analyzed by Western blotting analysis with a Myc antibody (upper panel). Data are presented as means \pm SD for threefive independent experiments $\left({ }^{*} \mathrm{P}<0.05\right.$, versus cells treated with or without TPA)

In the last decade, some studies focused on the post-translational modifications of PKB including ubiquitination [20]. For example, 17-(allylamino)-17-demethoxygeldanamycin( 17-AAG), an inhibitor of HSP90, induced cell apoptosis of the MCF-7 cell line, which was associated with the ubiquitination of $\mathrm{PKB}$, targeting it to the proteasome [21]. Wu et al. reported that the Sin1/mTORC2-mediated phosphorylation of PKB HM site targets PKB for K48-linked polyubiquitination and rapid proteasomal degradation
[22]. These results are consistent with our present study. It has been well documented that phosphorylation of numerous cellular targets, including serine/ /threonine kinases, initiates the ubiquitination-dependent degradation [22]. Previous studies showed that lack of PKB Thr450 phosphorylation in Sin1-deficient mouse embryonic fibroblast (MEF) cells caused PKB ubiquitination and degradation [23]. PKB phosphorylated at Thr308 could be ubiquitinated and targeted for proteasomal degradation [24]. Considering our 
A
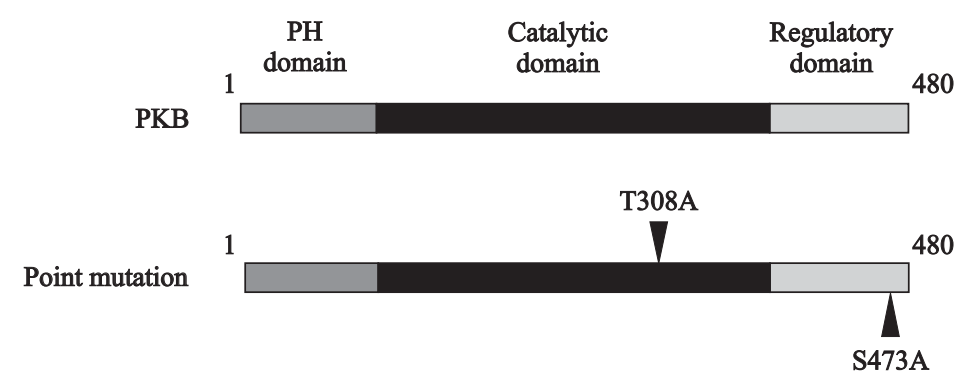

B

$\begin{array}{lllll}\text { TPA (h) } & 0 & 12 & 24 & 48\end{array}$
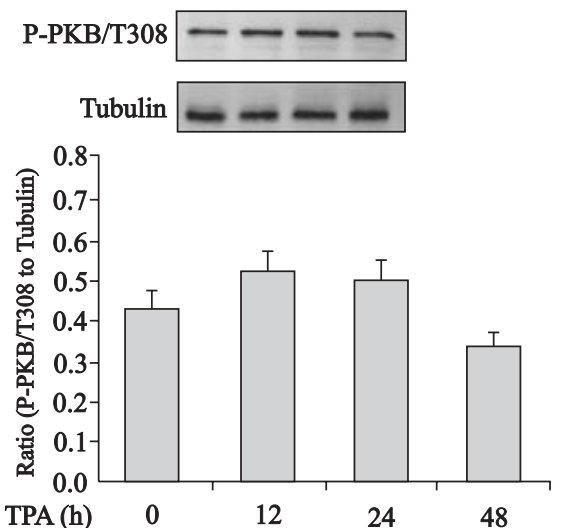

C

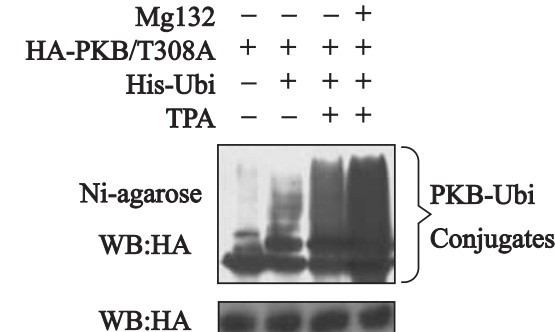

WB:HA

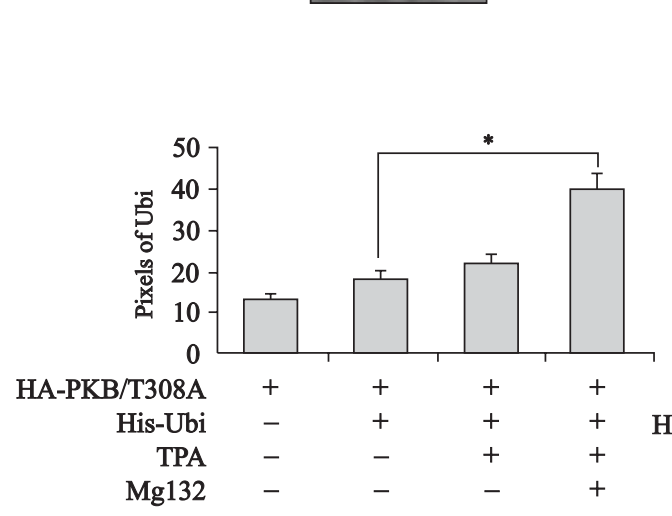

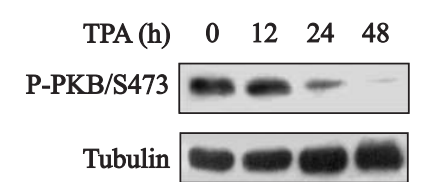
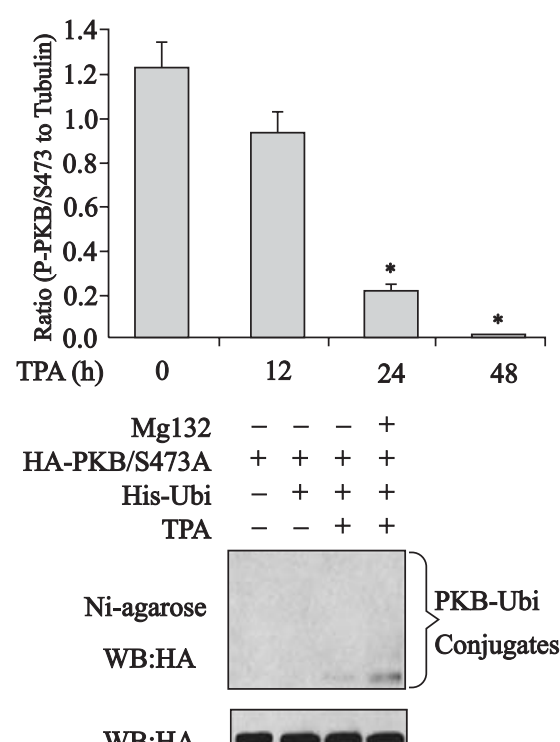

WB:HA

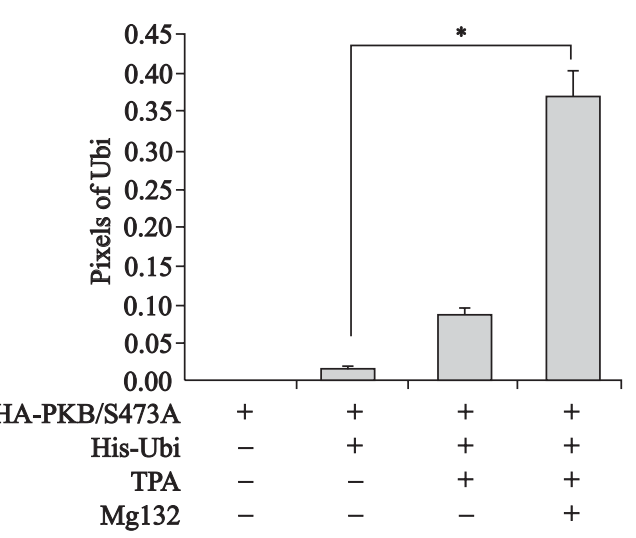

Figure 2. The ubiquitin-proteasomal pathway of PKB degradation induced by TPA is associated with its phosphorylation. A. Schematic representation of PKB point mutants. B. Effect of TPA on phosphorylation of PKB at Ser473 and Thr308 in gastric cancer BGC-823 cells. Cells were treated with TPA $(100 \mathrm{ng} / \mathrm{mL})$ for different time as indicated. Cell lysates were prepared as described in Materials and Methods, and then analyzed by Western blotting with anti-Phospho-PKB/Ser473 or anti-Phospho-PKB/Thr308 antibodies. Tubulin expression served as loading control. C. Effect of TPA on ubiquitination of PKB/T308A and PKB/S473A in HEK293T cells. Cells were co-transfected with HA-PKB/T308A or HA-PKB/S473A, and His-ubiquitin expression vectors. After transfection for $32 \mathrm{~h}$, cells were treated with MG132 (20 mM) for $3 \mathrm{~h}$ and/or TPA (100 ng/mL) for $24 \mathrm{~h}$. Cells lysates were prepared as described in Materials and Methods. A fraction of the lysate was analyzed by Western blotting analysis with a HA antibody (lower panel). The remainder of the lysate was incubated with Ni-agarose and bound proteins were analyzed by Western blotting analysis with a HA antibody (upper panel). Data are presented as means \pm SD for three-five independent experiments $(* \mathrm{P}<0.05$, versus cells treated with or without TPA) 
previous result showing that TPA caused a decrease of PKB activity by inhibiting its phosphorylation at Ser473 [4], we focused on the role of PKB Ser473 and Thr308 phosphorylation in its ubiquitination. In this report, we showed that TPA-induced inhibition of phosphorylation at Ser473 of PKB was the prerequisite for PKB ubiquitination, and PKB ubiquitination was dependent on the repression of phosphorylation of PKB Ser473, not Thr308. Wu et al. also found that the PKB Ser473 phosphorylation was involved in PKB ubiquitination, as it promoted a K48-linked polyubiquitination of $\mathrm{PKB}$, resulting in its rapid proteasomal degradation [22]. Therefore, phosphorylation of the Ser473 site indeed participates in PKB ubiquitination and degradation, even though it may play different roles in different cell lines. Taken together, these results support the view that PKB utilizes phosphorylation-dependent ubiquitination and degradation as a potential mechanism to terminate its activity, including Thr308, Thr450 and Ser473 phosphorylations.

Although the phosphorylation of Thr308 of PKB and Ser473 of PKB are prerequisites to PKB activation, it seems unlikely that TPA co-regulated these two processes. We observed that abolishing PKB Ser473 phosphorylation, but not PKB Thr308 phosphorylation, following TPA and MG132 stimulation blocked the ubiquitination of PKB. In fact, some reports demonstrated that extracellular factors have different effects on the two sites. For example, UCN01(7-hydroxystaurosporine) induced clinical insulin resistance by blocking PKB activation and subsequent GLUT4 translocation in response to insulin, and this effect appeared to occur by inhibiting Thr308 phosphorylation even though Ser473 phosphorylation was almost completely unaffected [25]. In WEHI-164 cells, tumor necrosis factor $\alpha$ (TNF- $\alpha$ ) activation of $\mathrm{PKB}$ was accompanied by increased phosphorylation of Ser473, but not Thr308 [26]. Li et al. showed that TPA reduced PKB phosphorylation only at Ser473 in mouse keratinocytes [27]. These studies indicate that in some cell lines extracellular factors do not necessarily affect the two PKB phosphorylation sites simultaneously. Accordingly, the effect of TPA on BGC823 cells occurred mainly through inhibiting PKB Ser473 phosphorylation to facilitate its ubiquitination, causing the degradation of PKB. Recently, Wu et al. proposed a model illustrating the mechanism of $\mathrm{PKB}$ regulation, in which PKB Ser473 phosphorylation may be targeted as an effective strategy to turn off PKB activity, thus marking phosphorylated Ser473 as an important PKB activation marker [22]. Our results have provided evidence supporting their argument. PKB activity regulation associated with the ubiquit- in-proteasomal pathway may shed new light on our understanding of human diseases and reveal novel strategies for therapy.

In conclusion, TPA could induce the degradation of $\mathrm{PKB}$ protein via the ubiquitin-proteasomal pathway, and the repression of PKB phosphorylation at the serine 473 site might be a prerequisite for its ubiquitination in gastric cancer cells.

\section{Acknowledgements}

We would like to thank Dr. Qiao Wu (Xiamen University, Xiamen, Fujian, China) for the generous supply of PKB expression vectors. The National Natural Science Foundation of China (No.81072015), the Natural Science Foundation of Fujian, China (No. 2010D007) and the Medical Innovation Foundation of Fujian, China (No. 2011-CXB-36) supported this study.

\section{References}

1. Okudaira N, Goto M, Yanobu-Takanashi R et al. Involvement of retrotransposition of long interspersed nucleotide element-1 in skin tumorigenesis induced by 7,12dimethylbenz[a] anthracene and 12-O-tetradecanoylphorbol13-acetate. Cancer Sci. 2011;102:2000-2006.

2. Wang F, Fu X, Chen X, Chen X, ZhaoY. Mitochondrial uncoupling inhibits 533 mitochondrial translocation in TPA-challenged skin epidermal JB6 cells. PLoS One. 2010;5: e13459.

3. Von Burstin VA, Xiao L, Kazanietz MG. Bryostatin 1 inhibits phorbol ester-induced apoptosis in prostate cancer cells by differentially modulating protein kinase $\mathrm{C}$ (PKC) delta translocation and preventing PKCdelta-mediated release of tumor necrosis factor-alpha. Mol Pharmacol. 2010;78:325-332.

4. Zhang B, Xia C. The expression of protein kinase B in gastric cancer cell apoptosis induced by 12-O-tetradecanoylphorbol-1, 3-acetate. Cell Mol Biol Lett. 2009;14:466-480.

5. Restuccia DF, Hemmings BA. From man to mouse and back again: advances in defining tumor AKTivities in vivo. Dis Model Mech. 2010;3:705-720.

6. Vanhaesebroeck B, Alessi DR. The PI3k-PDK1 connection: more than just a road to PKB. Biochem J. 2000;346:561-576.

7. Toker A, Newton AC. Akt/protein kinase B is regulated by autophosphorylation at the hypothetical PDK-2 site. J Biol Chem. 2000;275:8271-8274.

8. Manning BD, Cantley LC. AKT/PKB signaling: navigating downstream. Cell.2007;129:1261-1274.

9. Engelman JA. Targeting PI3K signalling in cancer: Opportunities, challenges and limitations. Nat Rev Cancer. 2009;9:550-562.

10. O'Reilly KE, Rojo F, She QB et al. mTOR inhibition induces upstream receptor tyrosine kinase signaling and activates Akt. Cancer Res. 2006;66:1500-1508.

11. Garcia-Echeverria C, Sellers WR. Drug discovery approaches targeting the PI3K/Akt pathway in cancer. Oncogene 2008;27:5511-5526.

12. Zunder ER, Knight ZA, Houseman BT, Apsel B, Shokat KM. Discovery of drug-resistant and drug-sensitizing mutations in the oncogenic PI3K isoform p110 alpha. Cancer Cell. 2008;14:180-192. 
13. Chen J, Tang H, Hay N, Xu, J, Ye RD. Akt isoforms differentially regulate neutrophil functions. Blood. 2010;115:42374246.

14. Pickart CM. Mechanisms underlying ubiquitination. Annu Rev Biochem. 2001;70:503-533.

15. Rui L, Fisher TL, Thomas J, White MF. Regulation of insulin/insulin-like growth factor-1 signaling by proteasome-mediated degradation of insulin receptor substrate-2. J Biol Chem. 2001;276:40362-40367.

16. Yang WL, Wang J, Chan $\mathrm{CH}$ et al. The E3 ligase TRAF6 regulates Akt ubiquitination and activation. Science. 2009;325:1134-1138.

17. Wu Q, Lin XF, Ye XF et al. Ubiquitinated or sumoylated retinoic acid receptor alpha determines its characteristic and interacting model with retinoid $\mathrm{X}$ receptor alpha in gastric and breast cancer cells. J Mol Endocrinol. 2004; 32:595-613.

18. Chen Claudia, Okayama Hiroto. High-Efficiency Transformation of Mammalian Cells by Plasmid DNA. Molec Cel Biol.1987; 7: 2745-2752.

19. Jaffray EG, Hay RT. Detection of modification by ubiquitinlike proteins. Methods. 2006; 38:35-38.

20. Yang WL, Wu CY, Wu J, Lin HK. Regulation of Akt signaling activation by ubiquitination. Cell Cycle. 2010; 9:487-497.

21. Basso AD, Solit DB, Chiosis G, Giri B, Tsichlis P, Rosen N. Akt forms an intracellular complex with heat shock protein
90 (Hsp90) and Cdc37 and is destabilized by inhibitors of Hsp90 function. J Biol Chem. 2002;277:39858-39866.

22. Wu YT, Ouyang W, Lazorchak AS, Liu D, Shen HM, Su B. mTOR complex 2 targets Akt for proteasomal degradation via phosphorylation at the hydrophobic motif. J Biol Chem. 2011;286:14190-14198.

23. Facchinetti V, Ouyang W, Wei H et al. The mammalian target of rapamycin complex 2 controls folding and stability of Akt and protein kinase C. Embo J. 2008;27:1932-1943.

24. Suizu F, Hiramuki Y, Okumura F, et al. The E3 ligase TTC3 facilitates ubiquitination and degradation of phosphorylated Akt. Dev Cell. 2009; 17: 800-810.

25. Kondapaka SB, Zarnowski M, Yver DR, Sausville EA, Cushman SW. 7-hydroxystaurosporine (UCN-01) inhibition of Akt Thr308 but not Ser 473 phosphorylation: a basis for decreased insulin-stimulated glucose transport. Clin Cancer Res. 2004;10:7192-7198.

26. O’toole A, Moule SK, Lockyer PJ, Halestrap AP. Tumor necrosis factor-alpha activation of protein kinase B in WEHI164 cells is accompanied by increased phosphorylation of Ser473, but not Thr308. Biochem J. 2001;359:119-127.

27. Li LW, Sampat K, Hu N, Zakari J, Yuspa SH. Protein kinase Cnegatively regulates Akt activity and modifies UCV-induced apoptosis in mouse keratinocytes. J Biol Chem. 2006; 281: 3237-3243. 